Cahiers $d u$ MONDE RUSSE

\section{Cahiers du monde russe}

Russie - Empire russe - Union soviétique et États indépendants

$60 / 4 \mid 2019$

Varia

\title{
Tom CUBBIN, Soviet Critical Design. Senezh Studio and the Communist Surround
}

\section{Natalia Prikhodko}

\section{OpenEdition}

\section{Journals}

Édition électronique

URL : https://journals.openedition.org/monderusse/11541

DOI : 10.4000/monderusse. 11541

ISSN : $1777-5388$

\section{Éditeur}

Éditions de l'EHESS

\section{Édition imprimée}

Date de publication : 1 octobre 2019

Pagination : 867-871

ISBN : 978-2-7132-2797-4

ISSN : 1252-6576

\section{Référence électronique}

Natalia Prikhodko, «Tom CUBBIN, Soviet Critical Design. Senezh Studio and the Communist Surround », Cahiers du monde russe [En ligne], 60/4 | 2019, mis en ligne le 01 octobre 2019, consulté le 08 janvier 2023. URL : http://journals.openedition.org/monderusse/11541 ; DOI : https://doi.org/ 10.4000/monderusse. 11541 


\section{Tom CUBBIN}

\section{Soviet Critical Design}

\section{Senezh Studio and the Communist Surround}

Londres - Oxford Bloomsbury (Visual Arts), 2018, 226 p.

Dédié au phénomène de l'atelier de Senež, le livre de Tom Cubbin réussit à exposer sa singularité en croisant plusieurs histoires et problématiques. Une histoire des idées et des concepts, une histoire des rencontres et des échanges humains, une histoire de la création et de la production en l'URSS sont abordées à travers l'étude de quelques projets emblématiques de l'atelier de Senež. Sans chercher à proposer une vision complète à aucune de ces histoires, Cubbin se concentre plutôt sur les façons dont elles s'interconnectent et se déterminent réciproquement afin de faire surgir la notion du design critique soviétique.

L'Atelier central d'éducation et d'expérimentation de l'Union des artistes de l'URSS, ou l'atelier de Senež, appelé ainsi car ses séminaires se tenaient dans une maison de création située près du lac de Senež, est un fruit de l'époque du dégel marquée par une réorientation idéologique et économique. L'émergence d'un discours favorisant la consommation accorde une place importante au design dans la production soviétique. Né d'une opportunité ouverte par ce discours, l'atelier de Senež ne s'intéresse pourtant pas aux bénéfices du consumérisme. Son objectif est de concevoir des environnements qui permettent une interconnexion harmonieuse entre l'homme, la nature, la culture, la technologie et une information orientée vers la création d'une culture de design authentiquement communiste. 
Cubbin propose le terme communist surround pour désigner un type d'environnement qui crée des formes de réception et d'échange d'information et un rapport à l'espace tels que, tout en répondant à un certain idéal communiste, ils stimulent la créativité dans les relations humaines, dans les attitudes au travail et dans l'interaction avec le monde matériel. L'évolution du concept de communiste surround détermine la focale de l'analyse de l'activité de l'atelier de Senež ainsi que le choix des projets étudiés dans le livre.

Parmi ceux-ci, nous noterons une Machine informatique domestique, dont le système de contrôle et de gestion d'information à la maison permet de créer son propre « environnement informatique subjectif »; un projet de bêtatron, qui favorise l'exploration des usages et les fonctionnalités multiples ; un Centre culturel souterrain, qui entraîne le visiteur dans un " labyrinthe de savoirs » composé des fragments de la civilisation européenne en forme d'images, de textes, mais surtout d'expériences qui invitent chacun à produire ses propres liens entre ces éléments ; ou encore des projets d'environnements urbains, qui permettent de conserver des lieux historiques au milieu d'ensembles modernistes et d'en faire la référence de l'identité locale, comme le projet pour le centre-ville de Neberežnye Čelny.

Cubbin étudie ces projets et s'intéresse particulièrement à leur dimension critique. Celle-ci réside dans la capacité à imaginer des manières d'interagir avec les environnements matériels et informatiques telles, qu'elles puissent produire les conditions nécessaires à l'émergence de personnalités harmonieuses, non aliénées, actives au sein de formes de vie et de travail collectives leur permettant de mettre en œuvre leur créativité. Cet idéal communiste, qui guide les recherches de l'atelier, fait également adopter un regard critique envers les pratiques du présent - que ce soit la réalité soviétique ou la réalité occidentale avec leurs rapports propres à l'information, à l'espace, à la production et la consommation des biens. Cette critique se manifeste dans la conscience projective (proektnoe soznanie), c'est-à-dire dans l'élaboration de projets pour l'avenir qui puissent à la fois engendrer des modèles de communication, de travail, de vie sociale alternatifs à ceux qui dominent dans le présent et être basés sur eux.

L'auteur explore les projets de l'atelier de Senež en tant que design critique et les croise avec des approches et des concepts développés dans d'autres pays. Il retrace les liens entre les membres de l'atelier de Senež, ses fondateurs Evgenij Rozenbljum et Karl Kantor en premier lieu, et des figures tels Tomás Maldonado, designer à l'École de design d'Ulm, Charles et Ray Eames, qui ont notamment conçu un dispositif de projection documentaire pour l'Exposition américaine de 1959 à Moscou, ou encore Oskar Hansen, architecte polonais et auteur d'un manifeste de la forme ouverte. À partir de ces croisements, l'ouvrage met en lumière certains parallèles conceptuels et valeurs communes qui surgissent dans le design critique sous des régimes politiques différents. Le terme communist surround est de ce point de vue emblématique, car il est choisi par l'auteur en résonance avec le terme démocratic surround employé par Fred Turner dans son analyse du design américain d'après-guerre. 
L'auteur inscrit les méthodes et les aspirations de l'atelier de Senež dans une histoire internationale du design critique et souligne aussi son ancrage complexe dans la culture soviétique qui touche à ses aspects multiples. D'une part, Cubbin met en lumière le contexte formé par le discours officiel sur la « révolution scientifique et technique » et par le développement, encouragé par la politique de l'État, de disciplines comme la cybernétique, l'ergonomie, l'ingénierie, la sociologie. De l'autre, il montre l'influence des théories qui ne trouvent pas toujours beaucoup de soutien dans les structures institutionnelles, mais qui nourrissent considérablement la réflexion et l'activité des milieux intellectuels. Il étudie plus précisément les influences du productivisme, notamment des textes de B. Arvatov et A. Toporkov, et celles de la philosophie méthodologique dont certains des auteurs, comme G. Ščedrovitskij ou O. Genisaretskij, ont donné des conférences lors de séminaires de l'atelier de Senež.

En plus des appuis théoriques, idéologiques et disciplinaires, le livre donne à comprendre comment les idées et les projets de l'atelier de Senež sont liés aux pratiques sociales propres aux milieux intellectuels à l'époque du socialisme tardif. L'auteur aborde notamment les notions de communication ou d'interaction (obščenie) et de milieu ou d'environnement (sreda) qui se réfèrent aux intérêts, attitudes, lieux mis en commun et partagés dans un cercle de personnes, créant ainsi un environnement vital pour la réalisation intellectuelle, professionnelle et personnelle.

Pour révéler la singularité du design critique développé au sein de l'atelier de Senež, Cubbin accorde une place importante à l'analyse de la terminologie soviétique relative au design. Design n'est pas un terme beaucoup utilisé en URSS et, pour désigner leur activité, les membres de l'atelier emploient celui de hudožestvennoe proektirovanie (conception artistique des projets, ou artistic projecteering dans la traduction anglaise de Cubbin). Ce terme se distingue des termes hudožestvennoe konstruirovanie [construction artistique] et oformlenie [mise en forme] ou encore de tehničeskaja estetika [esthétique technique], associé principalement à l'activité du VNIITE (Institut de recherche scientifique sur l'esthétique technique), un organisme d'État qui centralisait le design industriel et dont l'objectif était d'améliorer la qualité des objets destinés à la consommation de masse.

L'étude de la terminologie révèle les opérations concrètes et les composantes esthétiques, techniques et sociales qui se trouvent au cœur de la pratique du design. L'accent mis sur une composante ou une autre détermine l'affiliation institutionnelle et, par conséquent, le système de gestion et de contrôle. C'est ainsi que d'être affilié à l'Union des artistes avec des projets assez techniques et appliqués permet à l'atelier de Senež d'avoir une certaine liberté de création. D'une part, les projets ne sont pas soumis, comme dans les structures dirigées par les ingénieurs, à la pression des commandes d'entreprises ni à celle de répondre à une utilité immédiate. De l'autre, certaines formes plastiques, dont l'abstraction ou l'art cinétique qui sont exclues des expositions des beaux-arts, se trouvent ici tout à fait acceptées et justifiées en tant qu'études indispensables portant sur la forme, la couleur, la composition, la perception, etc. 
Cette liberté émerge donc des interstices institutionnels et disciplinaires. Elle donne lieu à des formes de création singulières qui échappent aux catégories qui, par exemple, structurent la culture occidentale. Ainsi, l'ouvrage de Cubbin éclaire certaines strates de la culture soviétique qui n'ont pas d'équivalent ailleurs et, dès lors, il ouvre un grand potentiel de questionnements sur les manières dont les phénomènes artistiques et intellectuels sont « rangés » et sur leur statut au sein des systèmes culturels.

Le livre propose des articulations analytiques subtiles et des observations attentives aux ambiguïtés de la culture soviétique, cependant, il laisse le lecteur insatisfait sous quelques aspects. Tout d'abord, la mauvaise qualité des images, en noir et blanc et souvent floues, empêche de saisir les détails des projets complexes discutés dans le livre. Ces illustrations sont pourtant essentielles pour appréhender et imaginer le traitement novateur de la forme et de la spatialité développé par l'atelier. Ensuite, le rapport entre projets réalisés et projets non-réalisés n'est pas assez clairement exposé. Bien que l'étude ne prétende pas à l'exhaustivité, il serait néanmoins important de comprendre la répartition du travail nécessaire entre la conception artistique du projet et sa réalisation concrète, complète ou partielle. Une telle articulation permettrait de mieux se rendre compte des conditions dans lesquelles une recherche critique a pu être menée au sein du système soviétique. Enfin, et pour la même raison, le rapport à l'idéologie soviétique aurait pu être davantage développé.

De manière plus générale, l'approche de Cubbin sur le rôle de l'idéologie présente à la fois une force et une faiblesse. Certes, l'auteur n'enferme pas son analyse dans les catégories idéologiques et les rapports aux structures du pouvoir soviétique, piège dans lequel se retrouvent encore certains écrits sur la culture soviétique. Il examine le discours idéologique et son évolution comme un conditionnement important parmi d'autres. Et c'est ainsi qu'il réussit à révéler naturellement la part de liberté de création et de conception qui était justement propre au système socialiste, en l'absence des contraintes de l'économie du marché. Cependant, en marginalisant le problème de la pression idéologique, cette discussion ne permet pas toujours d'apprécier par quel usage précis des règles et des relations professionnelles et personnelles ni par quelles négociations, explicites ou implicites, l'atelier de Senež a pu exercer son activité pendant trois décennies, entre 1964 et 1992, c'est-à-dire développer des projets critiques et, en même temps, recevoir des commandes de l'État, travailler à la fois avec les acteurs institutionnels et avec ceux de la culture dite « non officielle».

Avec des ouvertures révélatrices et en dépit de quelques lacunes sensibles dans son étude historique, l'ouvrage présente un grand intérêt du point de vue des idées et des concepts qu'il nous dévoile. Élaborés au sein de l'atelier de Senež à différentes périodes de son existence, ils paraissent extraordinairement actuels. Ainsi, une des objets d'études fondamentaux pris en compte dans les recherches de l'atelier concerne les conditions du travail créatif et collectif ainsi que les liens entre l'environnement, les méthodes et les produits du travail. La durabilité matérielle et esthétique des produits constitue un autre axe de réflexion important, qui 
incite notamment à développer des recherches sur la compatibilité et la coexistence harmonieuse entre les objets nouveaux et les objets du passé. Le principe central qui se trouve à la base de la méthodologie de l'atelier est celui de la forme ouverte. Pensée comme un système dynamique d'éléments, la forme ouverte se constitue à partir du corps du travailleur ou de l'usager qui peut la modifier et l'adapter facilement en fonction de son confort et de ses besoins : elle place l'homme au cœur de sa composition et lui permet d'imaginer une multitude de manières d'opérer possibles plutôt que de lui réserver une ou quelques fonctions fixes et prédéterminées. Les projets de l'atelier mettent aussi en scène le principe de la théâtralisation de l'espace urbain, qui pose notamment la question des rôles réservés aux habitants par les codes de comportement générés par cet espace ; le concept d'écologie culturelle, lui, interroge l'éthique de la production culturelle et ses interconnexions avec les formes de vie non humaines. Développées à partir d'un certain idéal communiste qui a nourri l'activité de l'atelier, ces idées et approches surprennent par leur résonance avec les débats et les besoins d'aujourd'hui et montrent qu'elles n'ont rien perdu de leur capacité à porter une conscience projective.

Natalia Prikhodko

CERCEC, EHESS 